\title{
Two distinct energetic electron populations of different origin in the Earth's magnetotail: a Cluster case study
}

\author{
I. I. Vogiatzis ${ }^{1,2}$, T. A. Fritz ${ }^{1}$, Q.-G. Zong ${ }^{1}$, and E. T. Sarris ${ }^{2}$ \\ ${ }^{1}$ Center for Space Physics, Department of Astronomy, Boston University, Boston, MA, USA \\ ${ }^{2}$ Space Research Laboratory, Dept. of Electrical and Computer Engineering, Democritus University of Thrace, Xanthi, Greece
}

Received: 21 December 2005 - Revised: 19 May 2006 - Accepted: 24 May 2006 - Published: 9 August 2006

\begin{abstract}
Energetic electrons $(\mathrm{E} \geq 30 \mathrm{keV})$ travelling along and perpendicular to the magnetic field lines have been observed in the magnetotail at $\mathrm{L} \sim 17: 00$ and 22:00 MLT during the recovery phase of a storm-time substorm on 7 October 2002. Three-dimensional electron distributions of the full unit sphere obtained from the IES/RAPID sensor system demonstrated a rather complicated and random behavior of the energetic electrons. Occasionally these electrons were appearing to travel parallel, perpendicular, or in both directions, relative to the magnetic field direction, forming in this way bi-directional, perpendicular-peaked, and mixed distributions. The electron enhancements occurred while the Cluster spacecraft were on closed field lines in the central plasma sheet approaching the neutral sheet from the northern tail lobe. Magnetic field and energetic particle measurements have been used from geosynchronous and Cluster satellites, in order to describe the general context of the event and then give a possible interpretation regarding the occurrence of the electron anisotropies observed by the IES/RAPID spectrometer on board Cluster. According to geosynchronous measurements an electron dispersionless ejection is very well correlated with a dipolar re-configuration of the magnetic field. The latter fact supports the idea that electrons and, in general, particle ejections at geosynchronous altitude are directly related to electric fields arising from field dipolarization caused by current disruption. Also, having as a main objective the understanding of the way 3-D electron distributions are formed, we have analyzed electron energy spectra along and perpendicular to the magnetic field direction, demonstrating the fact that the electron population consists of two distinct components acting independently and in a random manner relative to each other. This leads to the conclusion that these two electron populations along and perpendicular to the field are generated at different remote locations at
\end{abstract}

Correspondence to: I. I. Vogiatzis

(ivogiatz@ee.duth.gr) different rates. The main conclusion of the present paper is that the perpendicular-peaked electron enhancements (electrons with pitch angle around 90 degrees, subjected mainly to curvature drift) observed by Cluster are produced in a remote location duskward of the satellite location, due to the longitudinal and tailward expansion of a current disruption region, and subsequently transported to the Cluster location by means of curvature drift. On the other hand, bi-directional electrons (electrons with pitch angle around 0 and 180 degrees, bouncing mainly along the field lines) are believed to be generated in the vicinity of the neutral sheet or around an $\mathrm{X}$-type region, as suggested by a plethora of previous studies. Finally, in the Discussion section, we make an attempt to present in a more thorough way the substorm model developed by Vogiatzis et al. (2005), which is intimately related to the importance of X-line formation for the initiation of a substorm.

Keywords. Magnetospheric physics (Energetic particles, trapped; Magnetotail; Storms and substorms)

\section{Introduction}

One of the basic features associated with substorms is the appearance of supra-thermal particles in the near-Earth magnetotail (Baker, 1984). Plasma heating and particle acceleration is a longstanding issue in magnetospheric substorm physics. In the beginning of the satellite observations, it was reported that the energetic particles with several $100 \mathrm{keV}$ to $1 \mathrm{MeV}$ are often observed in the magnetotail, and it was suggested that the observed energetic particle bursts may be related to magnetic reconnection and the formation of a neutral line (Sarris et al., 1976; Sarris and Axford, 1979; Baker and Stone, 1976, 1977). Previous studies demonstrated that at radial distances $>10 R_{E}$ the electrons appear to be mostly isotropic, however, a number of events show field-aligned, bi-directional

Published by Copernicus GmbH on behalf of the European Geosciences Union. 
anisotropy. Hada et al. (1981) were the first to report systematic observations of bi-directional distributions. Based on IMP6 data they found such anisotropies between 10-30 $R_{E}$ in $\sim 10 \%$ of plasma sheet observations, preferentially in association with high $B$ elevation angles, i.e. in the central plasma sheet region. They concluded that the bi-directional electrons are produced by a Fermi-type acceleration process in the neutral sheet, where magnetic field lines have strong curvature and the electric field is anti-parallel to the direction of the electron curvature drift. Such field-aligned anisotropy could, for example, be provided by the magnetic reconnection, as shown in the kinetic simulations by Hoshino et al. (2001), where electron acceleration around the X-type region, followed by compression due to the reconnection outflow, produces supra-thermal, high-energy electrons. Bi-directional distributions were also observed by Smets et al. (1999) during substorm dipolarization at $\mathrm{L} \sim 11$, whereas at closer distances they were replaced by butterfly distributions (at $\mathrm{L} \sim 9$ ) or perpendicular-peaked distributions (at $\mathrm{L} \sim 7$ ). They modeled and explained such distributions as the result of largescale magnetic field dipolarization during substorms, having the conclusion that the (parallel) Fermi acceleration dominates at larger distances from the Earth compared to (perpendicular) betatron heating, which prevails at regions closer to the Earth. On the other hand, Klumpar et al. (1988), surveying a region covering all the local time and the geocentric distance from 6 to $8.8 R_{E}$, showed that highly collimated counter-streaming electrons at $\mathrm{keV}$ energies observed at the equatorial region are magnetically mapped to the highlatitude auroral ionosphere, having the greatest occurrence probability at $\sim 8 R_{E}$ around 23:00 MLT (Klumpar, 1993), which was taken to support the idea that these electrons are of auroral origin. In a recent study, Shiokawa et al. (2003) investigated the occurrence characteristics of the bi-directional electrons in the plasma sheet at $\left|X_{\mathrm{GSM}}\right|=9-19 R_{E}$ using data obtained by the AMPTE/IRM satellite. From the observed characteristics, they concluded that the major source of bidirectional electrons in the central plasma sheet lies in the vicinity of the neutral sheet, including Fermi-type acceleration, and the contribution from ionospheric electrons is minor in this tail region.

From above, it is clear that bi-directional electron anisotropies at radial distances beyond $10 R_{E}$ are mainly attributed to acceleration processes which are active in the vicinity of the neutral sheet. Although much work has been carried out regarding bi-directional electron distributions, almost nothing has been presented regarding the occurrence of perpendicular-peaked electron distributions in the mid-tail, which is something that has been addressed in a recent work by Vogiatzis et al. (2005). Their study was focused on energetic electrons peaked at 90-deg which were observed in the magnetotail at $\sim 19 R_{E}$ near local midnight during the recovery phase of a substorm event. Based on their observations, they provided evidences that a field dipolarization caused by current disruption and the associated particle acceleration re- gion, expanding both longitudinally and tailward, could account for the generation of the perpendicular-peaked electron distribution which subsequently appeared at the Cluster location by means of curvature drift. In association with the above, Williams et al. (1990), in a well studied substorm event, have shown that major electron flux increases were observed in the current sheet at energies $\geq 45 \mathrm{keV}$. They concluded that the increase in $\geq 45 \mathrm{keV}$ electron fluxes observed in the current sheet was inconsistent with an acceleration taking place locally. Instead, they suggested that these electrons may have been accelerated in an earlier local time sector due to inductive processes which took place during field dipolarization and subsequently drifted to the ISEE1 satellite.

In this paper, we analyze electron pitch angle distribution data acquired while the Cluster spacecraft were in the magnetotail at $\mathrm{L} \sim 17: 00$ and 22:00 MLT during the recovery phase of a storm-time substorm event on 7 October 2002. Analyzing the electron behavior along and perpendicular to the field, we were led to the conclusion that these two electron populations are acting independently and in a random way relative to each other, suggesting that are generated at different remote locations at different rates. The latter fact further supports the scenario that a tailward-azimuthally propagating current disruption front energizes the particles locally as it passes over them, generating in this way the 90-deg energetic electrons which are subsequently transported by means of curvature drift to the Cluster location. The above scenario provides a completely different acceleration mechanism than the one that is often invoked to explain the generation of the bi-directional electron population. Usually, these bi-directional electrons are related to an acceleration process (Fermi-type acceleration) which takes place in the vicinity of the neutral sheet.

\section{Observations and data analysis}

The present study is based on data acquired from the IES (Imaging Electron Spectrometer) sensor system, which consists of 3 heads, each one with a $60^{\circ}$ opening angle which is part of the RAPID (Research with Adaptive Particle Imaging Detectors) experiment on board Cluster (Wilken et al., 1997). The IES measures energetic electrons within the energy range $20-400 \mathrm{keV}$ and during the event was operating in burst mode. A description of how the data of this mode are displayed can be found in Vogiatzis et al. (2005). Also, together with the electrons, proton data of a 4-s time resolution are used, provided by the IIMS (Imaging Ion Mass Spectrometer) sensor system, which measures energetic ions within the energy range $10-1500 \mathrm{keV}$. The Cluster magnetic field measurements are provided from the FGM (Flux/Gate Magnetometer) instrument (Balogh et al., 1997), with a time resolution of $4 \mathrm{~s}$. In addition, concurrent measurements of energetic particle and magnetic field data were used from GOES8, GOES10, and LANL spacecraft, in order to have 
an overall view of the particle and magnetic field dynamical behavior during the substorm evolution.

On 7 October 2002 signatures of a storm-time magnetospheric substorm event were observed by a number of Earthorbiting spacecraft, with the $D_{s t}$ index reaching values below $-100 \mathrm{nT}$ between 07:00 and 08:00 UT. The peak amplitude of the substorm in question was about $-1500 \mathrm{nT}$, based on the Kyoto AL index. The average spatial positions of all spacecraft used in this study for the time interval 07:0009:30 UT are shown in Fig. 1. The Cluster constellation was located in the duskward magnetospheric sector, with the average spatial coordinates having values $\mathrm{X} \approx-14 R_{E}, \mathrm{Y} \approx 8 R_{E}$ and $\mathrm{Z} \approx 1 R_{E}$ in GSM coordinates, taking as a reference $\mathrm{SC} 1$. The satellites were approaching the equatorial plane from the north, with SC3 leading the rest of the satellites on their traverse from the northern to southern lobe.

Figure 2 gives an overview of proton and electron flux measurements obtained from geosynchronous and Cluster spacecraft, together with magnetic field data from the FGM experiment and plasma beta parameter from $\mathrm{SC}$, for the time interval 06:00-10:00 UT on 7 October 2002. Panels (a) and (b) show energy-integrated fluxes from the RAPID experiment. Panel (c) shows differential fluxes of energetic electrons from the 1991-080/LANL91 Los Alamos satellite, where there is a clear dispersionless electron ejection at $\sim 07: 41$ UT associated with the substorm onset. In panels (d) and (e) the Cluster magnetic field components $B_{x}$ and $B_{z}$ are shown in GSM coordinates. In order to distinguish different tail regions, such as lobe and plasma sheet, we have plotted plasma beta in panel (f). Based on the RAPID/proton and FGM/magnetic field data, the Cluster spacecraft were initially inside the plasma sheet, with the field having a highly stretched configuration. Eventually, the plasma sheet appeared to thin, letting the satellites enter into a nearly lobelike environment at $\sim 07: 44$ UT, where plasma beta showed a clear dropout. After 08:00 UT all four Cluster spacecraft were observing proton/electron bursts lasting for $\sim 25 \mathrm{~min}$ and then at $\sim 08: 28 \mathrm{UT}$, they finally re-entered the plasma sheet. During the whole time interval after 07:30 UT, $B_{x}$ was decreasing and at $\sim 09: 00$ UT it obtained relatively low values while $B_{z}$ was increasing, reaching values around $20 \mathrm{nT}$, aspects that are characteristic for the central plasma sheet region. Moreover, proton fluxes after the plasma sheet expansion returned to about the same level they had before the dropout, unlike the electron fluxes which showed a clear gradual enhancement during the recovery, obtaining their maximum value at $\sim 09: 15 \mathrm{UT}$.

Figure 3 gives 2-h intervals of GOES8 and GOES10 magnetic field measurements surrounding the event of interest. The data shown are of 1-min time resolution and are presented in the local PEN coordinate system, in which the $H_{p}$ component is parallel to the satellite spin axis, which is perpendicular to the satellite's orbital plane or parallel to the Earth's spin axis in the case of a zero degree inclination orbit. $H_{e}$ lies parallel to the satellite-Earth center line and points

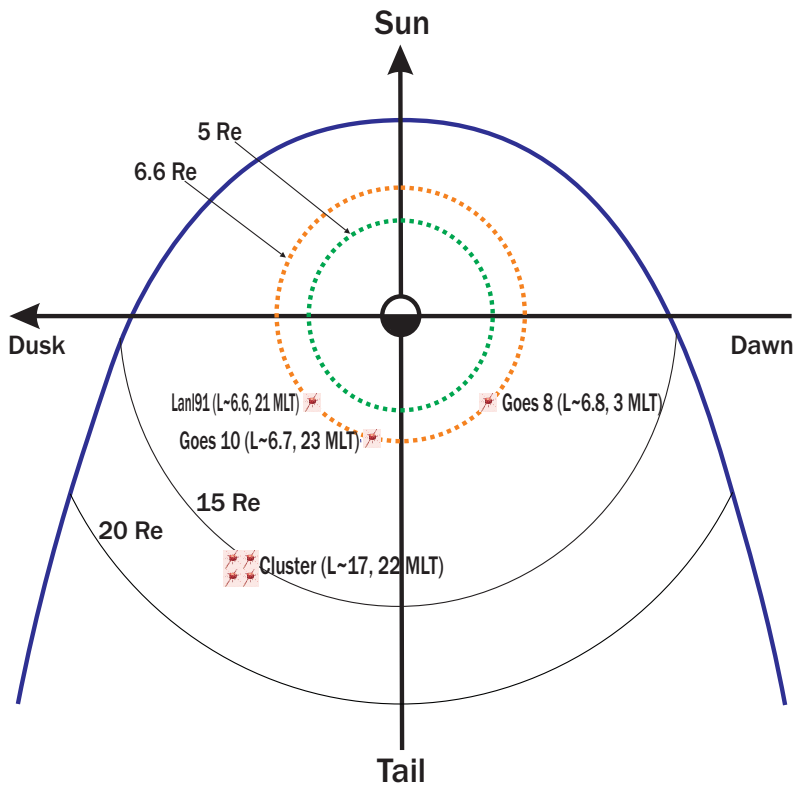

Fig. 1. The average spatial positions of all spacecraft used in this study for the time interval 07:00-09:30 UT.

earthward. $H_{n}$ is perpendicular to both $H_{p}$ and $H_{e}$, and points eastward. The most obvious changes in GOES10 data are the field dipolarizations at $\sim 07: 15$ and $\sim 07: 39$ UT. Prior to $07: 15 \mathrm{UT}$, the magnetic field had a relatively stretched configuration, as indicated by both $H_{p}$ and $H_{e}$ magnetic field components, with the elevation angle of the magnetic field vector $\phi=\arctan \left(H_{p} / H_{e}\right)$ being around 26 degrees. Just at 07:15 UT the field started to become more dipole-like, as revealed by the increasing magnitude of the $H_{p}$ but only for a limited time of $\sim 19 \mathrm{~min}$, returning immediately after to a stretched configuration, again for a limited time of $\sim 5$ min. Certainly, the most prominent field dipolarization occurred at $\sim 07: 39$ UT, closely associated with the dispersionless electron ejection at 1991-080 geosynchronous satellite at $\sim 07: 41$ UT. In a similar fashion GOES8 observed a field dipolarization at $\sim 08: 04$ UT, having a more step-like time evolution. Note the time lag of $\sim 25 \mathrm{~min}$ between the GOES10 and GOES8 field dipolarizations. This can be attributed to the time that current disruption effects need to propagate azimuthally, in order to cover the four hours distance in local time that separates the two satellites.

The variable electron pitch angle distributions observed by Cluster during its neutral sheet approach are demonstrated in Fig. 4. Here we show representative 3-D intensity distributions from $\mathrm{SC} 1$ averaged over the first 4 energy channels $(30-120 \mathrm{keV})$ and over $30 \mathrm{~s}$. Black contours in each panel indicate the loci of $10,30,60,90,120,150$, and 170 pitch angle electrons. The most important aspects that we would like to mention here are the two time intervals, 08:44-08:49 UT in the red frame and 09:15-09:20 UT in the green frame, during which we have the dominance of a bi-directional and peaked 

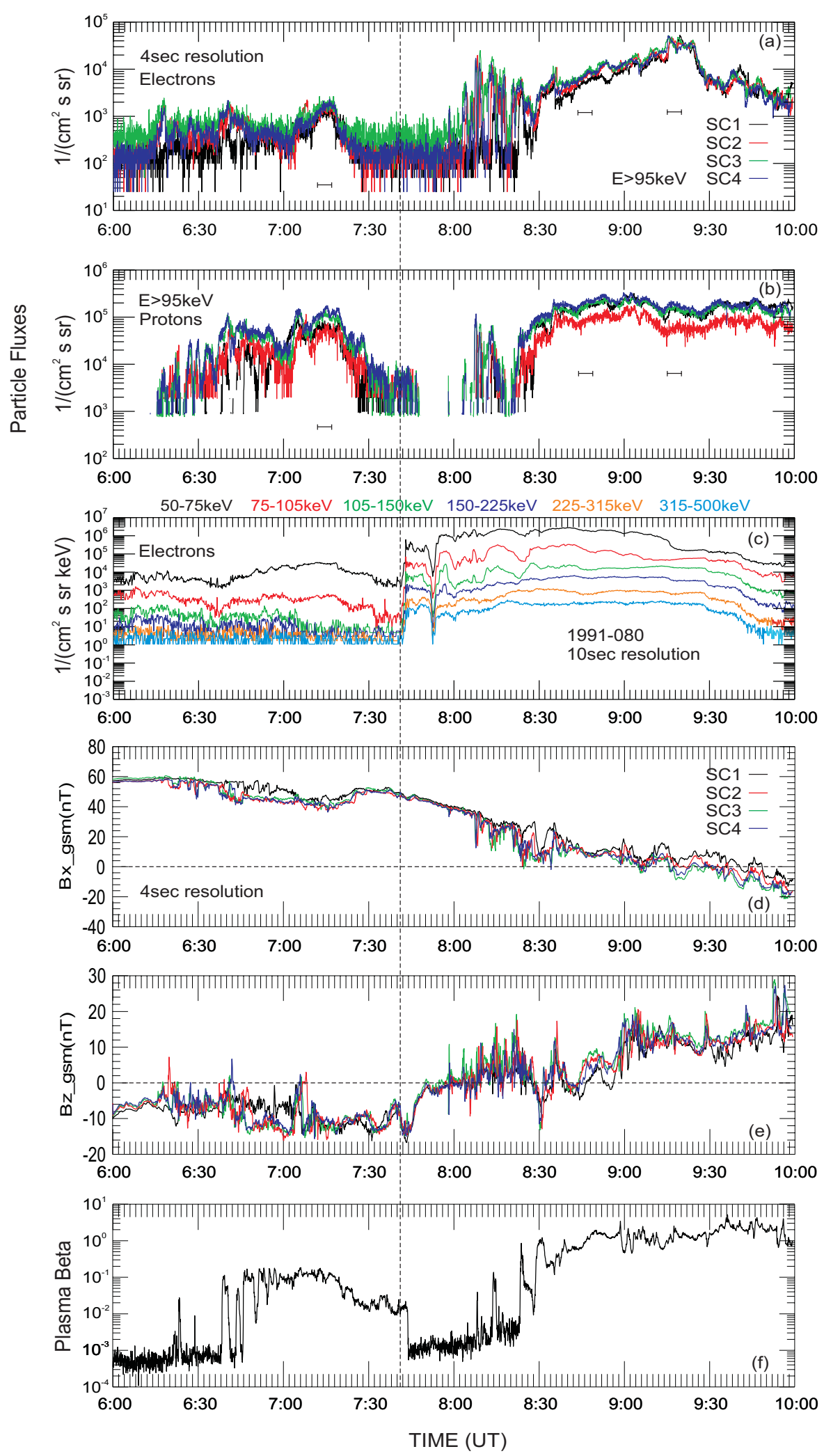

Fig. 2. An overview of proton and electron flux measurements (panels a-c) obtained from geosynchronous and Cluster spacecraft between 06:00-10:00 UT on 7 October 2002. The magnetic field components $B_{x}$ and $B_{z}$ are plotted in panels (d) and (e) in GSM coordinates. Plasma beta is plotted in panel (f), in order to distinguish between different tail regions, such as lobe and plasma sheet. Also, the horizontal bars denote 5-min time intervals for which proton and electron energy spectra have been calculated (see Fig. 5). 

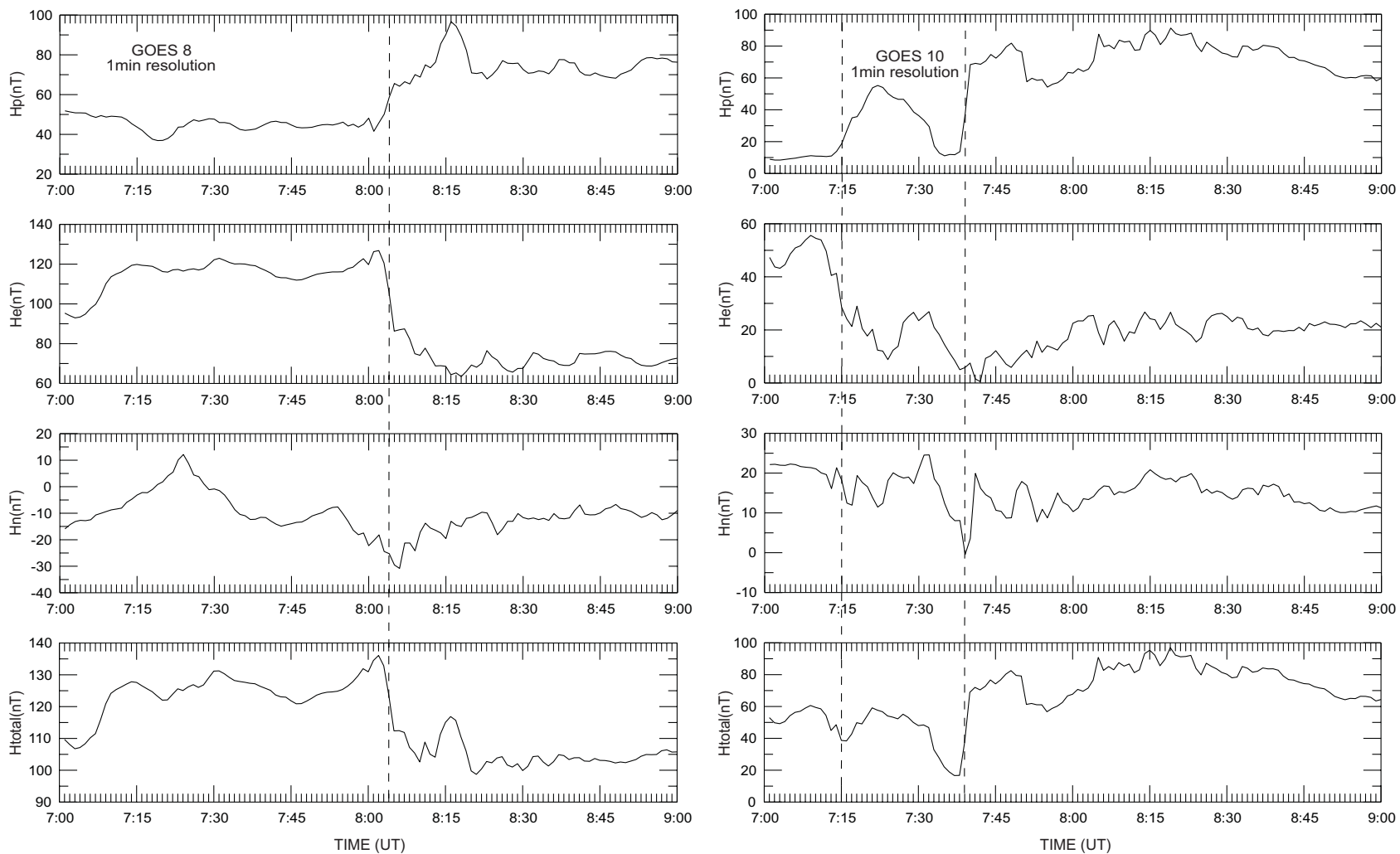

Fig. 3. GOES8 and GOES10 magnetic field measurements showing dipolarization signatures with the most prominent one at $\sim 07: 39$ UT.

90-deg distribution, respectively, and for which we have calculated the energy spectra (see Fig. 5). As we have already pointed out, during these time intervals the Cluster spacecraft were in the central plasma sheet approaching the neutral sheet from the north. Another noteworthy feature is the fact that during the second interval where we have the prevalence of the peaked at 90-deg distribution, the electron flux obtains its maximum value in all the Cluster spacecraft (Fig. 2, third horizontal bar in panel a). As it is clear, bi-directional (travelling along the field lines) and perpendicular-peaked (subjected to gradient-curvature drift) electrons are part of the central plasma sheet. The latter fact raises the question as to whether these two populations are related somehow or are they just two independent entities of the plasma sheet.

In Fig. 5 we show representative proton and electron energy spectra from $\mathrm{SC} 1$ for the three time intervals of $5 \mathrm{~min}$ in duration, each denoted by the horizontal bars in Fig. 2 . Note that in Fig. 5a we do not have any particular proton flux change during these intervals. This means that the proton population inside the plasma sheet remains almost unchanged during the magnetotail re-configuration. On the other hand, electrons (Fig. 5b) show clear flux enhancements in all energies during the magnetotail re-configuration.

The second time interval (red line) is characterized by the dominance of the bi-directional electron population, while during the third time interval (green line) we have the dom- inance of the perpendicular-peaked electron population. A conclusion that can be made is that the relative flux increase during the second interval can be attributed to the appearance of the bi-directional electrons, while the flux increase during the third interval can be attributed to the population drifting across the magnetotail perpendicular to the field lines.

In Fig. 6 we present electron time profiles along and perpendicular to the field direction for the first four energy channels (panels a-c). Panels (d), (e), (f) and (g) show the magnetic field components, $B_{x}$ and $B_{z}$, in GSM coordinates, the magnetic field elevation angle $\arctan \left(B_{z} / B_{x}\right)$ and the magnetic field magnitude $B_{\text {total }}$, respectively. What is shown in panel (h) is the dawn-to-dusk electric field $E_{y}$ in GSE coordinates. Note that there is no energy dispersion between the different energy channels, which is indicative that the event has already reached a steady state by the time we enter the plasma sheet, as we discuss later on. While the time profiles for the two pitch angle ranges along the field show similar behavior and seem to be well correlated, the electron population which is peaked at 90 -deg shows significantly different behavior. This fact provides us with a hint that the two energetic electron populations have different origins/sources and as a result, act independently relative to each other. An interesting feature that is revealed by examining panels (c), (d) and $(\mathrm{g})$ is the enhancement of the perpendicular flux starting at $\sim 09: 15$ UT, which coincides with a decrease in $B_{x}$ and 


\section{CLUSTER RAPID}

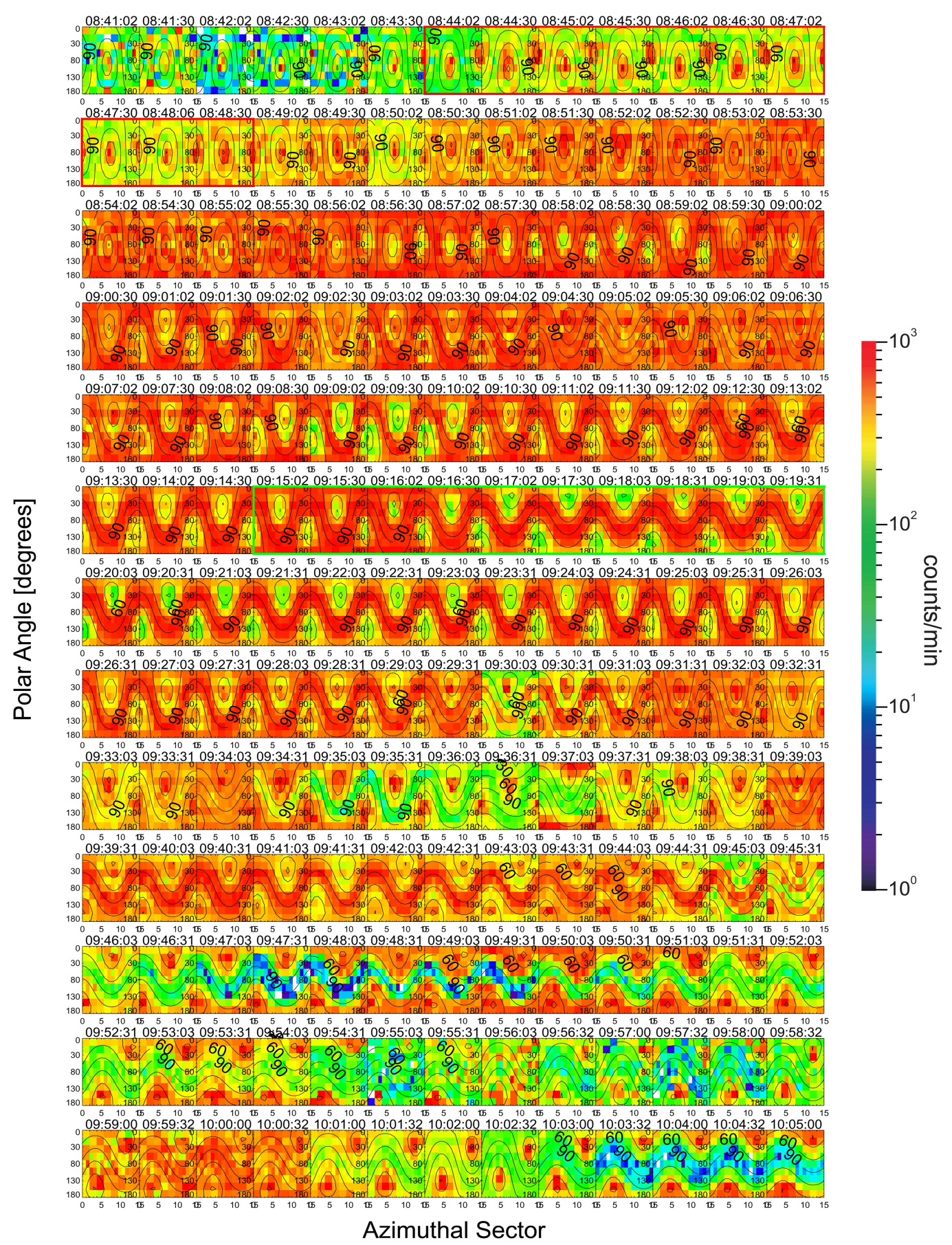

Fig. 4. Representative 3-D electron intensity distributions from SC1 for the energy range $30-120 \mathrm{keV}$. Superimposed are the different pitch angle contours. Note the transition from a clear bi-directional distribution to a mixed pitch angle distribution and then to a distribution showing the formation of field-aligned minima and so forth. Note, also, the red frames (in 1st and 2nd row) and the green frame (in the 6th row) which correspond to the 2nd and 3rd horizontal bars in Fig. 2 and denote 5-min time intervals where we have the dominance of a bi-directional and a perpendicular-peaked electron distribution, respectively. The abscissa of each 3-D plane projection corresponds to the 16 azimuthal sectors in which every spin is divided, with the 13th sector looking towards the Sun while the 9 polar look directions comprise the ordinate. 


\section{Spectra \\ 10/07/2002 \\ SC1}
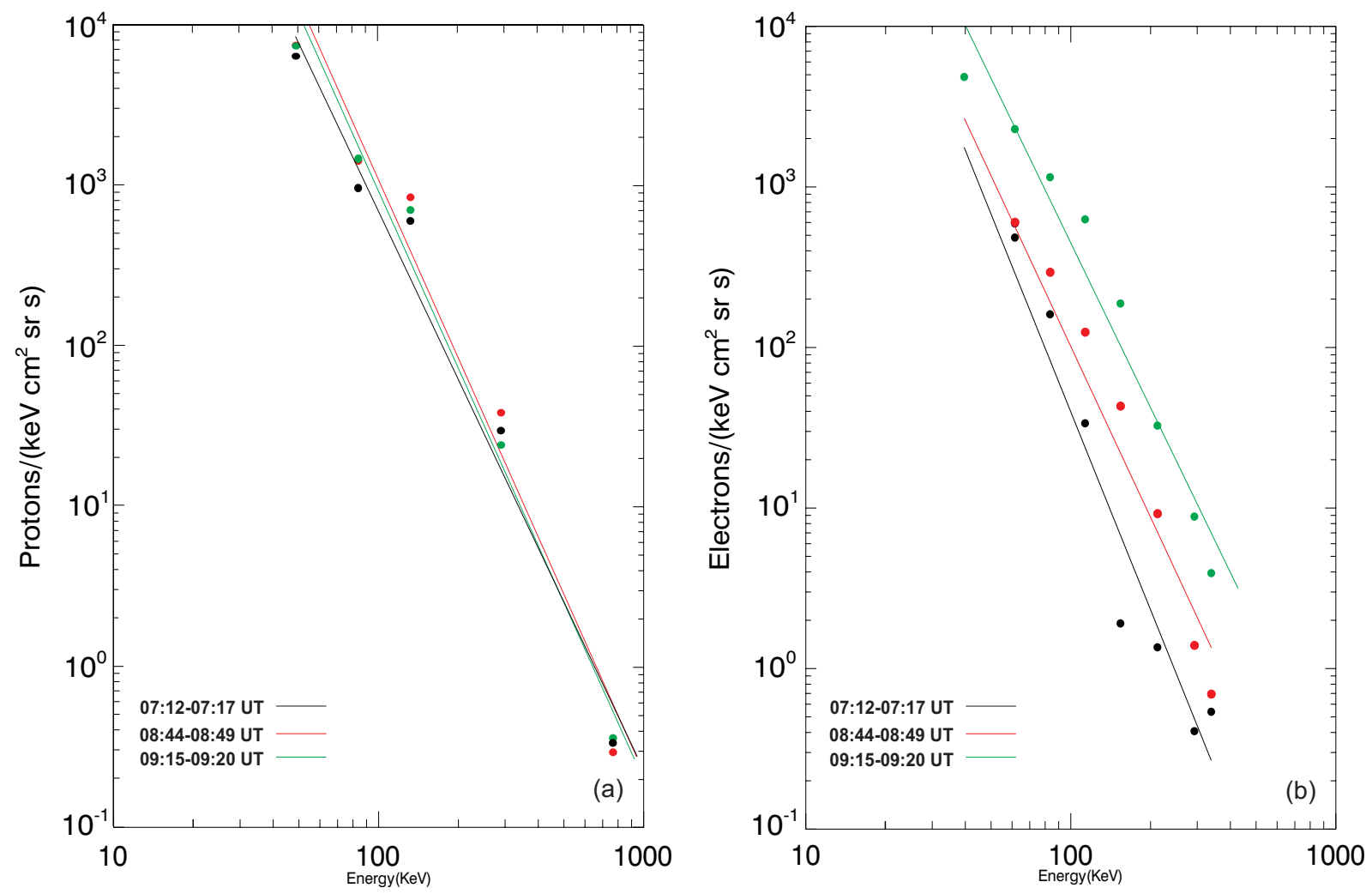

Fig. 5. Representative proton and electron energy spectra from SC1 for the intervals 07:12-07:17 UT (black color-coded), 08:44-08:49 UT (red color-coded) and 09:15-09:20 UT (green color-coded), during which the Cluster spacecraft were inside the plasma sheet (see panels (a) and (b) in Fig. 2). Also shown are the calculated best fits for the flux versus energy points. Unlike protons, electrons show successive, clear flux enhancements in all energies which are attributed firstly to the bi-directional and secondly to the perpendicular energetic electron population (red and green frames in Fig. 4).

$B_{\text {total }}$ (vertical dashed line). Also, as panel (f) indicates, the field is further dipolarized after this timing, which coincides with a nearly zero dawn-to-dusk electric field (panel h). Correspondingly, the enhancement of parallel flux at $\sim 08: 58 \mathrm{UT}$ also coincides with a moderate dipolarization and a zero duskward electric field.

Evidences are presented in Fig. 7 that the bi-directional electron population has no relation with the perpendicular one, but rather these two populations originate from different locations of the magnetosphere. In our analysis we have taken 2-min energy spectra samples, both parallel and perpendicular to the magnetic field direction. In panels (a), (c) and (e) we have chosen the bi-directional spectra samples in such a way so that they would not exhibit any essential changes, and compared them with the corresponding perpendicular ones, to examine if there exist any similarities in the spectral behavior of the two electron populations. As it is evident by comparing the panel pairs $(\mathrm{a}-\mathrm{b}),(\mathrm{c}-\mathrm{d})$, and $(\mathrm{e}-\mathrm{f})$, the two electron populations demonstrate a completely dif- ferent attitude, suggesting the lack of any connection between the two. Following the same procedure for the case now where the perpendicular spectra do not exhibit any essential changes, we are led to similar results (not shown).

The global magnetic field configuration just before the second dipolarization at GOES10 is presented in Fig. 8. What is shown is a schematic 3-D view of the magnetic field lines passing through the different satellites, depicting the magnetic field topology. Just before the field dipolarization, at $\sim 07: 39$ UT, the magnetic field is highly stretched, with relatively small elevation angles, while after the dipolarization phase onset and the propagation of the dipolarization front tailward, we start to have the substorm recovery phase. At $~ 09: 15$ UT, when the electron flux is maximized and the field-aligned minima have already formed, the magnetic field is already relaxed in a more dipolar configuration (see Table 1 for representative values of the elevation angle). 


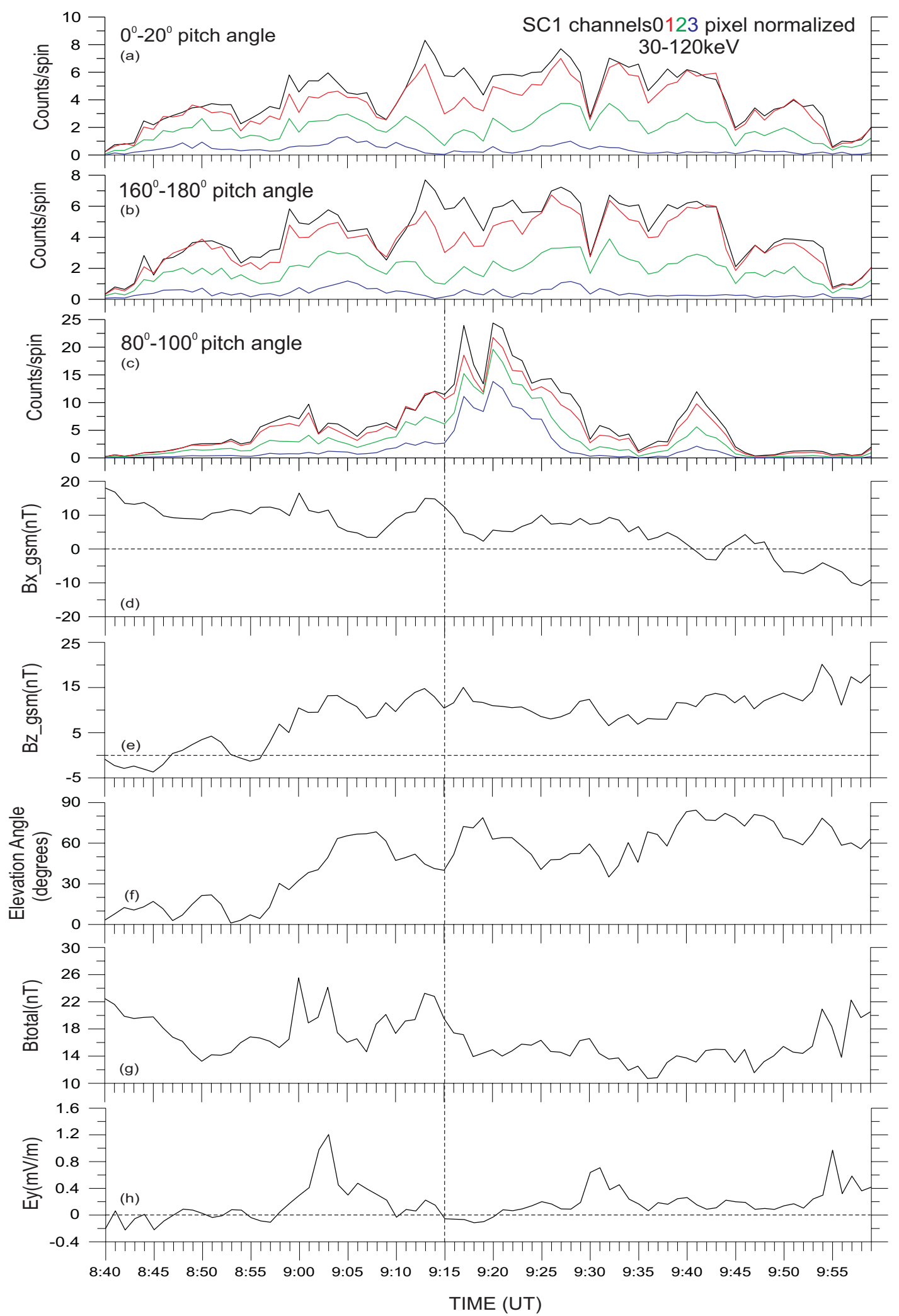

Fig. 6. Representative, 1-min time resolution measurements of energetic electron count rates, magnetic field parameters and the dawn-to-dusk electric field from SC1. In panels (a-c) the first four energy channels are shown in three different pitch angle ranges along and perpendicular to the field direction. Panels (d), (e), (f) and (g) show the magnetic field's $B_{x}, B_{z}$, elevation angle and magnitude, respectively. Also, in panel (h) the duskward electric field in GSE coordinates is shown. 


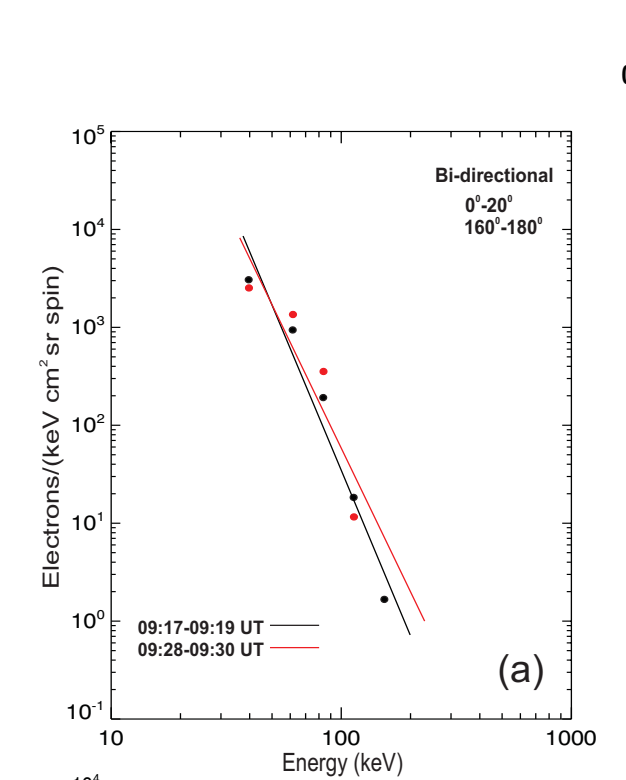

\section{Spectra \\ 07/10/2002 \\ SC1}
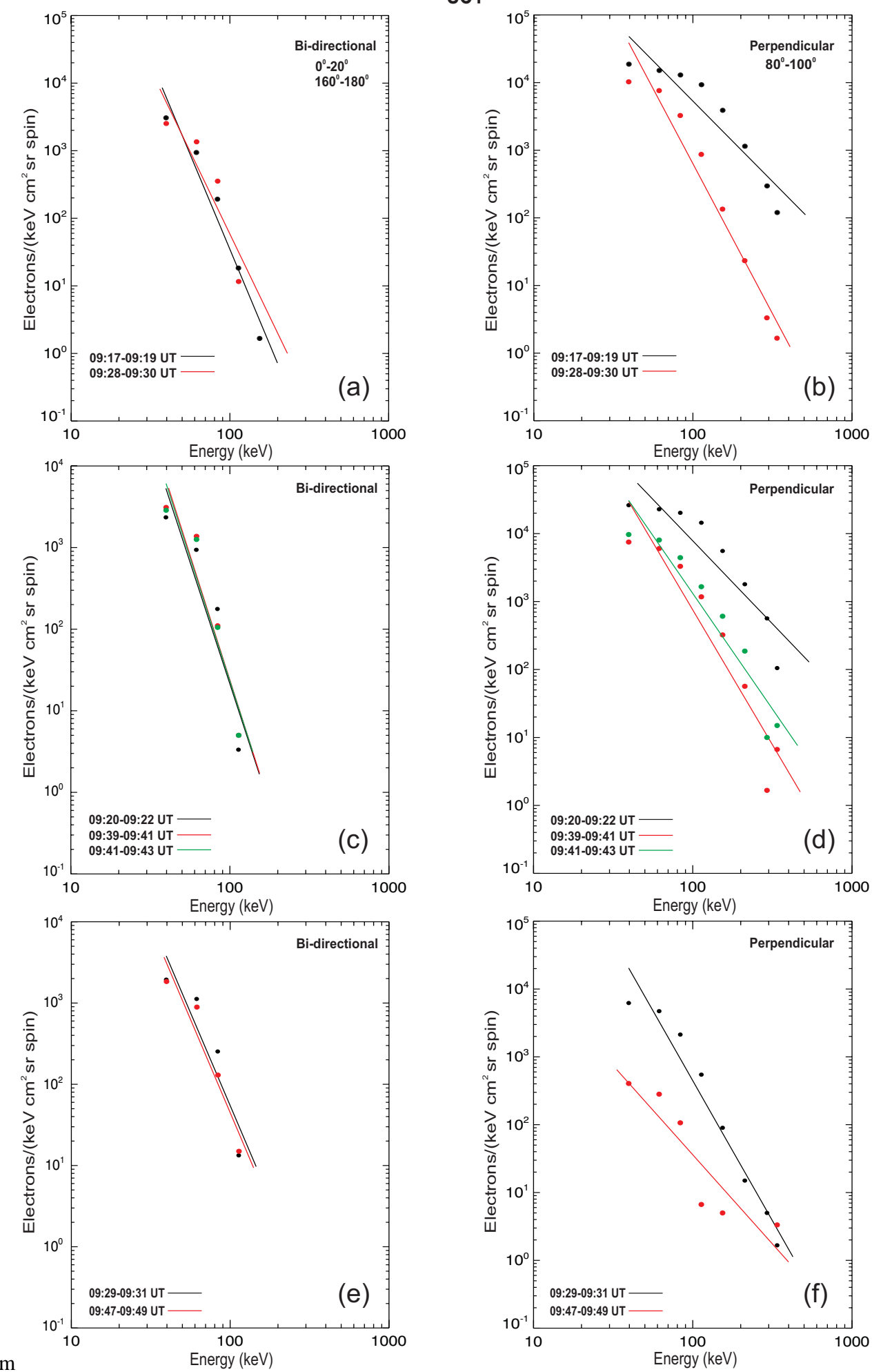

Fig. 7. Two-min energy spectra samples along and perpendicular to the field direction, demonstrating the independent behavior between the two energetic electron populations. 


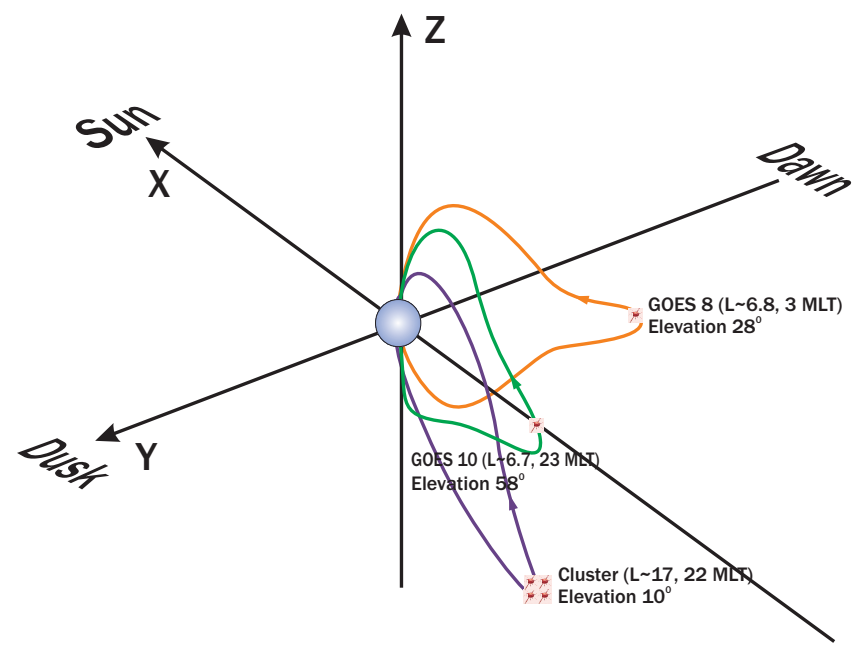

Fig. 8. Schematic magnetic field configuration just before the second dipolarization at GOES10 at $~ 07: 39$ UT, which is closely related to the dispersionless electron ejection observed by the 1991080 geosynchronous satellite at $\sim 07: 41$ UT. Also shown are the spacecraft positions and their magnetic field elevation angles.

\section{Interpretation}

As has been shown in Fig. 2 the substorm event under consideration was accompanied by intense particle ejections at geosynchronous altitude. By combining the observations from the geosynchronous satellites it appears that at $\sim 07: 39$ UT GOES10, which was located at 23:00 MLT, made an in-situ observation of the disruption of the cross-tail current associated with a dipolarization of the magnetic field (Takahashi et al., 1987), which, in turn, was intimately related to the ejection of electrons at LANL91. The two hours of local time separation that GOES10 and LANL91 have can account for the two minute time lag between the GOES10 field dipolarization and the dispersionless energetic electron ejection at the LANL91 location. These particle observations suggest that the magnetic field reconfiguration/variation was associated with a strong inductive electric field $\left(\partial B_{z} / \partial t\right)$ (Shepherd et al., 1980; Aggson et al., 1983) that energized the particles, an idea that is further supported by Lui et al. (1988).

As noted in the prior section, the first (minor) field dipolarization at GOES10 provides an indication that a pseudobreakup probably commenced at $\sim 07: 15$ UT, related to a partial cross-tail current disruption without developing into a full expansion phase, while the second field dipolarization marks the time of the substorm expansion effects passing from geosynchronous orbit, where the field is completely and irrevocably dipolarized. That a sustained disruption of the local cross-tail current and its diversion into the current wedge has taken place, is indicated by GOES 8 observations in Fig. 3. As we can see, the primary contribution to $H_{\text {total }}$ before the dipolarization at $\sim 08: 04 \mathrm{UT}$ is the addition of the
Table 1. Representative magnetic field elevation angles from GOES8, GOES10, and Cluster, where the relaxation of the magnetotail into a more dipolar configuration is clear as the time progresses.

\begin{tabular}{llll}
\hline UT & $\sim 07: 15$ & $\sim 07: 39$ & $\sim 09: 15$ \\
\hline GOES8 & $\sim 30 \mathrm{deg}$ & $\sim 28 \mathrm{deg}$ & $\sim 71 \mathrm{deg}$ \\
GOES10 & $\sim 26 \mathrm{deg}$ & $\sim 58 \mathrm{deg}$ & $\sim 72 \mathrm{deg}$ \\
Cluster & $\sim 19 \mathrm{deg}$ & $\sim 10 \mathrm{deg}$ & $\sim 65 \mathrm{deg}$ \\
\hline
\end{tabular}

$H_{e}$ component; thus, $H_{\text {total }}$ is positively correlated with the cross-tail current, $J$. Therefore, a disruption/diversion of $J$ will produce a decrease in $H_{\text {total }}$, which seems to be the case (Fig. 3, last panel in GOES8 observations) (see, for example, Fig. 5 in Vogiatzis et al., 2005, and Fig. 1 in Williams et al., 1990). Similarly to GOES8 observations, there is a good correlation between $H_{\text {total }}$ and $J$ also at the GOES10 location, until the time of the minor field dipolarization at $\sim 07: 15 \mathrm{UT}$.

By comparing the behavior of $H_{\text {total }}$ in GOES8 and GOES10 we can easily see that they behave in the opposite sense. While in GOES8, $H_{\text {total }}$, after the dipolarization, is reduced following the same pattern with $H_{e}, H_{\text {total }}$ in GOES10 is increased after both dipolarizations, being well correlated with the northward component $H_{p}$. The latter fact can be understood, if someone takes into account the four hour separation that the two satellites have in local time with GOES10 located at $\sim 23: 00$ MLT, which is regarded to be the average onset region from where local disruption of the crosstail current expands longitudinally with time, both eastward and westward, and the expansion results in the longitudinal propagation of substorm effects (Nagai, 1982; Lopez et al., 1988; Lopez and Lui, 1990). Furthermore, GOES10 is located very close to local midnight, a region whose magnetic field topology is expected to be influenced to a large extent by the transportation of mass, energy and northward directed magnetic flux (Angelopoulos et al., 1994, 1996), due to acceleration at a reconnection region (Baumjohann et al., 1990, 1999; Nagai et al., 1998). Often this magnetic flux being carried by fast plasma flows towards the Earth is considered to be the cause of flux pileup and field dipolarization near the geosynchronous orbit region (Hesse and Birn, 1991). In other words, the continuous accumulation of northward magnetic flux $H_{p}$ in the neutral sheet region plays the role of enhancing the northward magnetic flux of the background dipole magnetic field governing in this way the total magnetic field and producing the very good correlation between $H_{p}$ and $H_{\text {total }}$ that we see in Fig. 3. On the other hand, at GOES8 position, which is displaced significantly from local midnight, we would not expect any significant transportation of northward magnetic flux and as a result, the magnetic field configuration is intimately related to the behavior of the cross-tail current; thus, the radial component $H_{e}$ 
governs the total magnetic field $H_{\text {total }}$, something that is indeed observed based on Fig. 3. Summarizing the interpretation above it appears that both GOES8 and GOES10 made in-situ observations of the disruption of the cross-tail current associated with a dipolarization of the magnetic field. This cross-tail current disruption and field dipolarization is expected to progress tailward and to lead to plasma sheet expansion (Fig. 2, 08:28 UT and thereafter) and eventually signal the late stage of substorm recovery phase, where there will be a balance between magnetic tension and magnetic pressure (Vogiatzis et al., 2005).

By combining Figs. 2 and 5 it appears that the bidirectional electrons are part of the outer plasma sheet, whereas the 90-deg electrons make their appearance while approaching the current sheet region. The latter fact can be explained in terms of the different bouncing lengths that the two populations have, with the perpendicular electrons having the shorter lengths. Thus, as the plasma sheet expands, carrying with it the two anisotropic electron populations, the Cluster spacecraft eventually first intersect the bi-directional paths and then the 90-deg electron paths, suggesting that the event is spatial in nature. The fact that the proton spectrum remains almost unchanged, even after the plasma sheet expansion at $\sim 08: 28 \mathrm{UT}$, means that we do not have the addition of an extra proton population in the plasma sheet. This is in antithesis with the electrons, where there is obviously the clear enhancement of the electron spectrum during the second and the third time intervals, implying that the fluxes in all energies owe their existence first to the bi-directional and then to the 90-deg energetic electron population. The above means that the two energetic electron populations, along and perpendicular to the magnetic field direction, are essentially two fresh energetic electron components of the plasma sheet, which significantly enhance the pre-existing electron population.

The conclusion that the two electron populations are independently originating from different magnetospheric regions and that they are not the result of an acceleration mechanism taking place locally, is supported if we examine Fig. 6. By close inspection the following inferences can be made: a) the enhanced electron fluxes in both parallel and anti-parallel directions make up the highly-collimated bi-directional distribution. Since both flux levels are well balanced (nearly equal), it is suggested that the electron beams are bouncing between two mirror points on closed field lines throughout the whole period of observation. b) The comparison of the time profiles of the two electron distributions indicates that the bi-directional population does not owe its existence to the migration of electrons from the 90-deg pitch angle range and vice-versa. c) The good correlation between the different energy channels in both distributions suggests that the electron fluxes along and perpendicular to the field are not produced due to a shift from different energy channels, but rather originate from different regions of the magnetosphere following the dynamical behavior and the energization efficiency of their sources. As a result, energetic electrons appear simultaneously at all energies for all the pitch angle ranges with the different energy channels exhibiting the same time profiles. d) The fact that the perpendicular flux enhancement at $\sim 09: 15$ UT coincides with a decrease in magnetic field intensity provides strong evidence that the perpendicular acceleration of electrons is not related to a betatron acceleration through conservation of the first adiabatic invariant. Also, the associated field dipolarization, together with the lack of any electric field activity implies that the perpendicular flux enhancement is not related to any inductive effects taking place locally. Consequently, both conclusions support the idea that the event is not a temporal effect but rather has a spatial character.

Based on the above inferences and Fig. 7, which clearly demonstrates the independence and the randomness of the two electron distributions, one can be led to the reasonable conclusion that these two populations along and perpendicular to the field are generated at different remote locations at different rates with the complete lack of any connection between the two. This conclusion is further supported by Vogiatzis et al. (2005), who presents the scenario that a tailward-azimuthally propagating current disruption front can account for the generation of the 90-deg energetic electrons, which arrived at the location of Cluster by means of curvature drift. The latter fact comes in antithesis with the generation of the bi-directional electrons which are related to a neutral sheet acceleration mechanism.

\section{Discussion}

There are two possible mechanisms for producing perpendicular-peaked electrons. The first one is the betatron acceleration mechanism, where electrons gain energy in the perpendicular direction through the conservation of the first adiabatic invariant. This is achieved when the magnetic field strength increases slowly in time. Although $\mu$ remains constant the particle kinetic energy is changed due to the presence of electric fields and hence the perpendicular energy is increased due to the constancy of $\mu$. The other possible mechanism is the direct electron energization due to inductive electric fields, arising from magnetic field dipolarization. The second process likely produces energetic electrons in a wide range of pitch angles. The observations in the present study (Fig. 6) do not seem to be consistent with the production of the perpendicular-peaked electrons by betatron acceleration or by inductive electric fields taking place locally. On the contrary, the 90-deg electron profiles seem to behave independently relative to the magnetic or electric field changes.

On the other hand, as suggested by Hada et al. (1981); Smets et al. (1999); Shiokawa et al. (2003), a Fermi-type acceleration near the neutral sheet seems to be the major candidate for the production of the bi-directional electrons. As 
discussed by Shiokawa et al. (2003), to create the acceleration energy, it is necessary to consider a dawn-to-dusk electric field, which is equivalent to earthward plasma flow perpendicular to the northward magnetic field. However, based on panel (h) in Fig. 6, the duskward electric field during the time period examined exhibits relatively low values, with most of the time being around zero. The latter fact puts into question a Fermi-type acceleration process being active locally and producing the parallel flux enhancements. Rather, the event seems to be generated by a Fermi-type acceleration mechanism taking place in a remote location or being the result of an energization mechanism present around a remote $\mathrm{X}$-type region, as suggested by Hoshino et al. (2001).

Having as a motive the fact that the observations presented here support to a large extent the work by Vogiatzis et al. (2005), we try in the following paragraphs to substantiate in a more thorough way the substorm model developed in that study.

During the growth phase the magnetic flux contained in the magnetotail increases substantially. The plasma convection associated with the solar wind electric field mapped down into the open magnetosphere moves magnetic flux into the tail lobes. In a steady-state scenario magnetic reconnection in the neutral sheet prevents buildup of magnetic flux, but if for some reason this reconnection does not take place over some time period, or is not efficient enough, then the magnetic flux in the magnetotail eventually increases. The rate of increase of this tail flux is greatest during time periods when the solar wind dynamic pressure is high and when the IMF is southward, because in the latter case the dayside reconnection process is more efficient (Cravens, 1997). During this phase the magnetic field lines in the tail become more stretched and tail-like.

In the steady-state approach, where the time derivatives are set equal to zero, energization of particles is possible only if the flux of the Poynting vector $\frac{1}{\mu_{0}} \oint_{S}(\boldsymbol{E} \times \boldsymbol{B}) \cdot d \boldsymbol{a}$ (see Eq. 2), through a closed surface $S$ bounding our system, is negative, corresponding to electromagnetic energy inflow ( $d \boldsymbol{a}$ is always directed outwards). Adopting such an approach leads to an oversimplified treatment of the dynamical behavior of the phenomena taking place during substorms. This is especially true during the substorm expansion phase, which is characterized by large and rapid changes in the electric and magnetic fields and where the assumption of time independence is clearly inadequate (Cravens, 1997).

Based on the above considerations and due to observational reasons derived from a previous section (dispersionless particle ejection along with a prominent field dipolarization), there is an imperative theoretical need for inclusion of the inductive electric fields in any theory regarding the conversion of magnetic energy to particle kinetic energy. The best way to do that is by directly using Poynting's theorem, which relies on the fundamental principles of electromagnetic theory and it can be written as

$$
\begin{aligned}
\int_{V}(\boldsymbol{E} \cdot \boldsymbol{J}) d \tau= & -\frac{1}{\mu_{0}} \oint_{S}(\boldsymbol{E} \times \boldsymbol{B}) \cdot d \boldsymbol{a} \\
& -\int_{V}\left(\epsilon_{0} \boldsymbol{E} \cdot \frac{\partial \boldsymbol{E}}{\partial t}+\frac{1}{\mu_{0}} \boldsymbol{B} \cdot \frac{\partial \boldsymbol{B}}{\partial t}\right) d \tau \\
\frac{d W}{d t}=- & \frac{1}{\mu_{0}} \oint_{S}(\boldsymbol{E} \times \boldsymbol{B}) \cdot d \boldsymbol{a} \\
- & \frac{d}{d t} \int_{V} \frac{1}{2}\left(\epsilon_{0} E^{2}+\frac{1}{\mu_{0}} B^{2}\right) d \tau[\text { Joule/s }],
\end{aligned}
$$

where we integrate over the volume $V$ which is bounded by the closed surface $S$ (for the above formulation, see Griffiths, 1999). The first integral on the right-hand side reflects the energy rate transferred by the electromagnetic fields through the volume $V$ bounded by the surface $S$. The second integral is the total energy stored in the electromagnetic fields. The energy rate on the left-hand side represents the electromagnetic energy dissipation within the volume $V$. In other words, this term includes all the mechanic (kinetic) energy gained by charged particles (being within the volume $V$ ) from the electromagnetic fields.

With values typical for the magnetotail the ratio of magnetic to electric energy densities is $c^{2} B^{2} / E^{2}>>1$; hence, the magnetic energy prevails to a large extent against the electric energy. It is therefore legitimate to neglect the first term inside the volume integral on the right-hand side. As a result, the term

$$
\frac{1}{\mu_{0}} \boldsymbol{B} \cdot \frac{\partial \boldsymbol{B}}{\partial t}=\frac{\partial}{\partial t}\left(\frac{B^{2}}{2 \mu_{0}}\right)
$$

is the one describing the conversion of the internal energy (magnetic energy) stored in the system enclosed inside the volume $V$. The factor $\partial \boldsymbol{B} / \partial t$ represents the inductive electric field introduced by Faraday's law. Thus, it is clear that time dependence plays a vital role during the substorm expansion phase, where magnetic energy previously stored within the magnetotail constitutes an internal magnetospheric source for particle acceleration through the induction of electric fields.

\subsection{Poynting's theorem and GOES observations}

Having in mind the above considerations, it is interesting to examine again GOES8 and GOES10 magnetic field observations. If, for example, we take a closed surface $S$ encompassing the GOES8 satellite, we notice that during the field dipolarization at $\sim 08: 04$ UT the magnetic field magnitude decreases with time. Consequently, from Eq. (3) we have that $\frac{\partial}{\partial t}\left(\frac{B^{2}}{2 \mu_{0}}\right)<0$. Thus, this field reduction corresponds exactly to an internal magnetic energy dissipation process taking place within the volume $V$, which, in turn, is associated 
with a magnetic energy transformation into particle kinetic energy $\int_{V}(\boldsymbol{E} \cdot \boldsymbol{J}) d \tau>0$ (see Eq. 1) through the generation of inductive electric fields. In contrary to GOES8, GOES10 demonstrates a magnetic field magnitude increase. In order to understand the process leading to such a behavior, we have to take into consideration the fact that GOES10 is located very close to local midnight. The magnetic field topology of this region is expected to be influenced to a large extent by the transportation of mass, energy and northward directed magnetic flux (Angelopoulos et al., 1994, 1996), due to acceleration at a reconnection region (Baumjohann et al., 1990, 1999; Nagai et al., 1998), as we have already noted in the Interpretation section. Consequently, apart from the conversion of the magnetic energy stored locally, which is an internal process, we have additionally the transportation of magnetic energy from the space outside the volume $V$. This constitutes an external process where the Poynting vector flux through the closed surface $S$ is negative, $\frac{1}{\mu_{0}} \oint_{S}(\boldsymbol{E} \times \boldsymbol{B}) \cdot d \boldsymbol{a}<0$. Hence, during this process our system is supplemented with additional magnetic energy which counterbalances or even surmounts the local energy dissipation, leading to the observed field magnitude increase at GOES10 location. On the other hand, at GOES8 position, which is displaced significantly from local midnight, we would not expect any significant transportation of electromagnetic energy and as a result, the magnetic field magnitude to be governed by the internal energy dissipation, leading to its reduction. Although in the Interpretation section we followed a plasma dynamics approach to explain GOES observations, here we provide an alternative approach based on the principles of classical electrodynamics.

\subsection{Field dipolarization and inductive electric fields}

Now, if we take, for example, the cross section of the magnetotail north lobe, then due to plasma convection, a timevarying magnetic field will start to occur. This field change introduces, based on Faraday's law, an inductive electric field which tries to oppose the cause, thereby producing this field increase. Consequently, this electric field is producing work. The energy that is consumed to overcome this work originates from the solar wind and is exactly the magnetic energy which is stored in the lobe, thereby producing the stretchedlike configuration. As a consequence, a cross-tail current is built, carried by plasma sheet particles, primarily due to curvature drift and partly due to gradient drift, on closed field lines. This process constitutes a current generator, in which the current is the direct result of the kinetic energy of the plasma sheet particles. If the particle orbits are adiabatic, the loss cones will be nearly empty. An empty loss cone essentially decouples the magnetosphere from the ionosphere. As soon as the appropriate conditions are set in the current sheet, the kinetic cross-field streaming/current (KCSI/CFCI) instability (Lui et al., 1990, 1991) is initiated

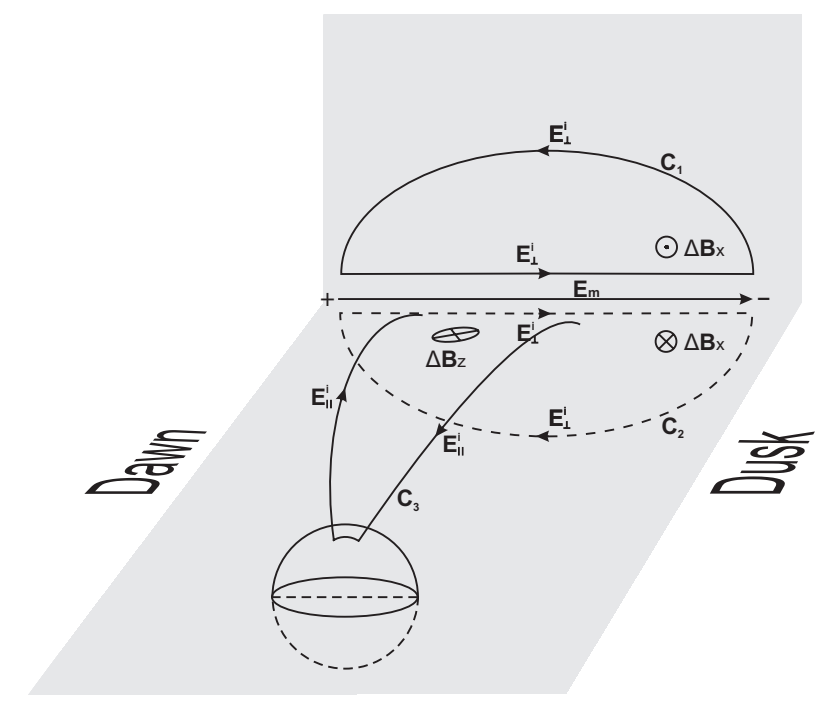

Fig. 9. A perspective drawing illustrating the electric fields induced during the progression of the cross-tail current disruption and the associated field dipolarization front tailward. Also shown are the magnetic field variations associated with these inductive electric fields. $\boldsymbol{E}_{m}$ denotes the steady-state magnetospheric (cross-tail) electric field associated with the plasma polarization.

(Vogiatzis et al., 2005). The associated wave-particle interactions cause an intense electron pitch angle scattering (Kennel and Petschek, 1966), distorting and subsequently diverting the electron cross-tail current that has been established during the late growth phase to the ionosphere, and this diversion is sufficient to initiate tail collapse, as suggested by Kaufmann (1987). The latter process has two consequences. The first is the field dipolarization associated with the induction of an electric field which energizes the particles (GOES10 observations in conjunction with the dispersionless electron ejection at Los Alamos satellite), while the second is the filling of the particle loss cones which couples the magnetospheric plasma to the ionosphere. Moreover, the inductive electric field is the primary agent for transforming previously stored magnetic energy into particle kinetic energy.

Choosing appropriate contours to enclose the regions where magnetic field dipolarization takes place, the variation of the magnetic flux going through these closed contours will introduce electromotive forces

$$
\oint_{C} \boldsymbol{E}^{i} \cdot d \boldsymbol{l}=\varepsilon=-\frac{d \Phi}{d t}
$$

associated with inductive electric fields. The work produced by these electric fields originates from the magnetic energy previously stored in the magnetotail during the growth phase which is now released and converted into particle energy. The direction of the inductive electric fields is such as to produce currents which will oppose the magnetic field variations and hence will try to reduce $B_{z}\left(H_{p}\right)$ and strengthen the $B_{x}$ 
$\left(H_{e}\right)$ magnetic field components. Hence, taking, for example, the $\mathrm{C}_{3}$ closed contour which follows the current wedge and is closing through the cross-tail direction (not shown in Fig. 9), we see that the direction of the induced electric field is such as to produce a $\Delta \boldsymbol{B}_{z}$ which will oppose a $\boldsymbol{B}_{z}$ increase. The same applies to contours $\mathrm{C}_{1}$ and $\mathrm{C}_{2}$, as well. The directions of the induced electric fields are shown in Fig. 9. The induced cross-tail electric field in $\mathrm{C}_{3}$ is directed duskward amplifying the electric field induced by the reduction in the $B_{x}$ component in both the north and the south lobe. At this time we have local current disruption and the appearance of the transient electric fields $\boldsymbol{E}_{\|}^{i}$ electrons obtain very large parallel velocities because of their large mobility. These energetic electrons constitute the connective link between the motion of the anti-sunward propagating disruption front and the ionosphere; a process which is directly related to the poleward expansion of the auroral electrojet and auroral luminosity (Akasofu et al., 1974; Rostoker et al., 1975; Shepherd et al., 1980; Jacquey et al., 1991). Consequently, the electron field-aligned current far exceeds the corresponding ion current. This charge delivery will have the result of reducing the impulsive $\boldsymbol{E}_{\|}^{i}$ and thus reinforcing $\boldsymbol{E}_{\perp}^{i}$ because the total integral

$\oint_{C_{3}}\left(\boldsymbol{E}_{\|}^{i}+\boldsymbol{E}_{\perp}^{i}\right) \cdot d \boldsymbol{l}=\epsilon=-\frac{d \Phi}{d t}$

around the closed circuit is not affected. Ions are not affected by the short-lived $\boldsymbol{E}_{\|}^{i}$ because of their large inertia. After the current disruption and the elimination of the parallel component $\boldsymbol{E}_{\|}^{i}$, the particles gyrate, bounce and gradientcurvature drift occurs in the already dipolarized magnetic field. It would be interesting here to emphasize that the rapid electron response and the subsequent elimination of the parallel inductive electric field can be viewed in terms of a magnetosphere considered to be filled with a highly conducting plasma, which yields the condition $\boldsymbol{E} \cdot \boldsymbol{B}=0$.

So, in summary, the inductive electric field during field dipolarization can be divided into two components; one parallel and one perpendicular to the magnetic field direction. The first is generated from a $B_{z}$ increase and is responsible for the intensification of FACs while the second is generated from both a $B_{z}$ increase and a $B_{x}$ decrease and its resultant has a dawn-to-dusk direction which is by far greater than the corresponding cross-tail electrostatic field component $\boldsymbol{E}_{m}$ (Heikkila and Pellinen, 1977; Aggson et al., 1983).

\subsection{Particle energization and non-adiabaticity}

Particles may become untrapped due to a violation of the first adiabatic invariant (particle's magnetic moment). The condition for violation of the first adiabatic invariant is that the particle sees either temporal field variations on the time scale of the gyroperiod, or spatial field variations on the order of the gyroradius.
Keeping in mind that above, it will be interesting at this point to discuss the possible mechanism responsible for the generation of the 90-deg energetic electrons associated with the expansion of field dipolarization front azimuthally and tailward (Vogiatzis et al., 2005). Ions execute an oscillatory motion about the plane of the current sheet, owing to the highly stretched magnetic field configuration (Speiser, 1965). Similarly, electrons due to the excitation of the kinetic instability will also behave non-adiabatically, executing an oscillatory motion about the plane of the current sheet. As we have already said in the previous subsection, this electron pitch angle scattering will have the effect of reducing the cross-tail current, thereby forcing the magnetic field to dipolarize. During dipolarization, which is on the time scale of a ion gyroperiod (Delcourt, 2002), the ion's nonadiabatic behavior will be further enhanced. Ions which behave non-adiabatically, owing to both spatial and temporal field variations, move towards dusk and electrons behaving non-adiabatically only due to temporal field variations move towards dawn, so that both ions and electrons in these oscillatory trajectories are continuously accelerated predominantly by the dawn-to-dusk inductive electric field across the geomagnetic tail. Moreover, due to the presence of a nonzero, northward magnetic field across the current sheet, particles are deflected towards the Earth (Speiser, 1965). Thus, during magnetic field dipolarization, particles can impulsively be energized in the current sheet by the application of a locally, short-lived inductive electric field, primarily in the perpendicular direction and then be ejected from the current sheet towards the Earth into the already dipolarized magnetic field (Delcourt et al., 1990; Delcourt and Moore, 1992; Delcourt and Sauvaud, 1994; Delcourt, 2002). At this point, however, it will be worthwhile to make a parenthesis and clarify that although in the present study we utilize an acceleration mechanism (magnetic field dipolarization associated with induced electric fields and violation of the first adiabatic invariant), which is similar to that presented in the previous cited studies, the physical view of magnetospheric substorm evolution that these authors adopt is completely different from ours. In the dipolarized field electrons conserve their magnetic moment, and hence they will return to a guiding center motion (West et al., 1978). On the contrary, ions in the supra-thermal energy regime are expected to behave in a non-adiabatic manner and hence their pitch angle distribution looses its anisotropic features. This follows from the fact that ions are subjected to a continuous isotropization because of random changes in the magnetic moment each time they cross the middle of the plasma sheet, experiencing a spatial variation of the magnetic field on the order of their gyroradius (collisionless pitch angle scattering) (Gray and Lee, 1982). The above mechanism further forces energetic ions to execute Speiser orbits and as a result, the ions return to the current sheet in a location much closer to the Earth than the one from which they were ejected. 
Magnetic field dipolarization can be considered as a selfpreserved process, acting in a feedback manner. With the initiation of the KCSI/CFCI instability discussed in Vogiatzis et al. (2005) the magnetic field starts to dipolarize, owing to cross-tail current reduction. Consequently, a dawn-todusk inductive electric field arises which accelerates unmagnetized ions in adjacent regions and leads to further spatial spreading of the unstable region in the neutral sheet (Lui et al., 1990). In the unstable region both ions and electrons again behave non-adiabatically, leading to consequences which we have already discussed.

The completely different particle attitude can explain the fact why electrons after the plasma sheet expansion are highly anisotropically peaked at 90 degrees, executing gradient-curvature drift in antithesis with protons which do not show any particular anisotropic behavior, with their flux levels showing almost no change during the re-enveloping of the Cluster spacecraft by the plasma sheet (see, for example, panel (b) in Fig. 2 and also Fig. 3, panels (g) and (h) in Vogiatzis et al., 2005). Inversely, the fact that we do not have any directional proton anisotropies inside the plasma sheet that would enhance the proton plasma sheet population similar to the electron case is indicative that energetic protons do indeed behave non-adiabatically when they cross the midplane of the plasma sheet, with the direct consequence of being isotropized via pitch angle diffusion.

In summary and in conjunction with Cluster observations, we could say that the absence of any directional proton anisotropy at the Cluster location can be thought of as the combination of two processes. The first process is related to the fact that protons are progressively isotropized during their traverse through the plasma sheet midplane while the second can be viewed as the consequence of the first one, that is, protons while executing Speiser orbits are displaced further earthward each time they come across the non-adiabatic region of the central plasma sheet. Yet the latter process is expected to persist in time until protons find themselves in an appropriate field topology with $B_{z}$ large enough so that the protons behave in an adiabatic manner.

It is noteworthy at this point to emphasize the triple role of the inductive cross-tail electric field $\boldsymbol{E}_{\perp}^{i}$ during field dipolarization, which is (a) to accelerate dramatically (from hundreds of $\mathrm{eV}$ up to hundreds of $\mathrm{keV}$ ) both ions and electrons (Delcourt et al., 1990) before they are ejected out of the current sheet towards the Earth. The above serves to enhance energetic particle populations earthward of the tailward-azimuthally expanding field dipolarization front; (b) to produce impulsive (up to a few hundreds of $\mathrm{km} / \mathrm{s}$ ) $\boldsymbol{E} \times \boldsymbol{B}$ bulk plasma earthward flows which are comparable to the velocity of supra-thermal ions (Delcourt et al., 1990). This role is analogous to that of the dawn-to-dusk convection electric field, which, on the one hand, accelerates the particles as they cross the current sheet while, on the other hand, produces earthward bulk plasma flows. Particle acceleration/impulsive bulk plasma convection, due to dipolarization associated in- ductive effects, can be viewed in terms of an internal process driven from the conversion of previously stored magnetic energy into particle-plasma kinetic energy, which is independent from any external system energy contribution (for instance, contribution from BBFs), and (c) to cause further violation of the first adiabatic invariant and reinforce in this manner the ion's pitch angle diffusion and equivalently nonadiabatic behavior. The latter process can be understood if we consider a particle in a rest frame fixed to the Earth, having an initial speed $v$ and pitch angle $\alpha$. It is possible to study the effects of the cross-tail inductive electric field by using Galilean transformation (for transformation velocities small compared to the speed of light). The moving frame, during the application of the $\boldsymbol{E}_{\perp}^{i}$, can then be considered to be moving earthward with speed $E_{\perp}^{i} / B_{z}$. In the moving frame the same particle has an initial speed $v^{\prime}$ and pitch angle $\alpha^{\prime}$. It is easy to see that the reference frames are related through the equations $v_{x}^{\prime}=v_{x}-E_{\perp}^{i} / B_{z}, v_{y}^{\prime}=v_{y}$ and $v_{z}^{\prime}=v_{z}$. By applying the transformation the speed and the pitch angle in the moving frame are given by

$v^{\prime}=\left\{\left[v_{x}-\left(\frac{E_{\perp}^{i}}{B_{z}}\right)\right]^{2}+v_{y}^{2}+v_{z}^{2}\right\}^{\frac{1}{2}}$

and

$\cos \left(\alpha^{\prime}\right)=\frac{\boldsymbol{v}^{\prime} \cdot \boldsymbol{B}}{\left\|\boldsymbol{v}^{\prime}\right\|\|\boldsymbol{B}\|}=\frac{\left[\boldsymbol{v}-\left(E_{\perp}^{i} / B_{z}\right) \hat{x}\right] \cdot \boldsymbol{B}}{\left\|\boldsymbol{v}^{\prime}\right\|\|\boldsymbol{B}\|}$,

where $\boldsymbol{v}=v_{x} \hat{x}+v_{y} \hat{y}+v_{z} \hat{z}$ and $v^{\prime}=\left\|\boldsymbol{v}^{\prime}\right\|$. The latter process is expected to affect mostly heavier ions, like $\mathrm{O}^{+}$and $\mathrm{He}^{+}$, owing to local gyroperiods of the order of the field variation/dipolarization time scale (Delcourt et al., 1990). The above treatment is an equivalent way of looking to the same process which is not other than magnetic field dipolarization whose time scale is comparable to the ion's gyroperiod.

The cross-tail current during the late stage of substorm growth phase is much more intense as we more closer to the Earth and maximizes at the transition region between the dipole and tail magnetic field configurations (Birn and Hesse, 1996; Birn et al., 1997b, 1998, 2000). We believe that this transition region most of the time is located inside a geosynchronous orbit at $\sim 4-5 R_{E}$ geocentric distance, while during extreme geomagnetic activity conditions, as those reported by Baker et al. (2004), the region moves even closer to the Earth. The fact that the cross-tail current directly reflects the magnetic field intensity in the lobes of the magnetotail supports the contention that during the late stage of substorm growth phase magnetic field energy storage maximizes at the transition region between the two field configurations and decreases as we proceed tailward. At the explosive onset of the expansion phase intense particle acceleration is taking place and the previously stored magnetic energy is converted to particle kinetic energy through the generation of inductive electric fields. The whole phenomenon is expected to diminish as field dipolarization caused by current disruption 
progresses tailward because of the continuously decreased magnetic energy stored in the system (evolution of substorm recovery phase).

4.4 Ring current enhancement and geosynchronous particle ejections

In the study by Vogiatzis et al. (2005) it has been shown that field dipolarization and associated particle acceleration not only expands longitudinally and propagates anti-sunward down the tail but also begins relatively close to the Earth, well inside geosynchronous orbit at $\mathrm{X} \approx 4.3 R_{E}$. A similar result has also been presented before by Friedel et al. (1996), who, based on CRRES satellite data, showed that dispersionless onsets can occur far into the inner magnetosphere down to $\mathrm{L} \sim 4.3$ and are distributed up to $\pm 5 \mathrm{~h}$ around local magnetic midnight. Having magnetic field dipolarization associated with cross-tail current disruption initiated inside geosynchronous altitude is something that has very important implications to the storm-time ring current. The ejection of energized equatorial plasma earthward into closed $\mathrm{L}$ shells while experiencing intense perpendicular energization and consequent trapping in the equatorial vicinity (Delcourt et al., 1990; Delcourt and Sauvaud, 1994; Delcourt, 2002), implies that the storm-time dipolarization of the magnetic field lines appears as an effective mechanism to populate the ring current, as suggested by in-situ measurements (Daglis and Axford, 1996; Daglis et al., 1999). Moreover, particle energies up to $1 \mathrm{MeV}$ or more, which are typical of the stormtime particles in the trapped radiation belts, can be produced with electric fields of the magnitude found in the magnetotail and also populate regions that are normally quiescent (Baker et al., 2004).

The fact that in geosynchronous orbit we occasionally observe dispersionless proton ejections preceding electron ejections and vice-versa, or simultaneous ejections of both species (Korth et al., 1991; Birn et al., 1997a; Vogiatzis et al., 2005), is a rather complex phenomenon. One factor that controls the process is how far earthward are the ions ejected when crossing the current sheet plane in a non-adiabatic manner. Energetic ions will behave adiabatically or not depends, to a large extent, on how much energy they gain before ejected from the current sheet and how much the magnetic field left after the cross-tail current disruption dipolarized is. Consequently, the proton's magnetic moment as a function of time depends on the energy after ejection and the degree of field curvature evolution after current disruption, assuming that electrons behave non-adiabatically only in the region where we have the kinetic instability excitation.

To obtain a sense of the substorm associated particle ejections it would be worthwhile to note the following: due to local field dipolarization we have the generation of a locally inductive electric field. This transient electric field is capable of accelerating the local plasma population up to suprathermal energies through the serpentine motion in the cur- rent sheet. In this manner the particles are energized and subsequently ejected earthward, migrating to already dipolarized magnetic field lines where they behave adiabatically. The presence of $B_{z}$ plays an important role in the acceleration process by controlling the time the particles spend in the current sheet before being ejected from it. As far as the appearance of geosynchronous particle ejections is concerned, the acceleration efficiency depends upon the transience of the field dipolarization, which produces the inductive electric field, which, in turn, is expected to depend on local time. Whether we observe only dispersionless electron ejection or only dispersionless proton ejection, or both of these ejections during substorm onset initiation, depends on several factors: a) the transience of field dipolarization producing the inductive electric field and how this relates to the violation of the proton and electron first adiabatic invariants; $b$ ) the position in local time of the satellite that makes the observation and the dependence on local time of the dipolarization process; $c$ ) the azimuthal extent of the current wedge at geosynchronous altitude. Two satellites only a few Earth radii apart would see very different effects if one is inside the wedge and the other is not; and d) the energy range of the pre-existing particle population. If this energy range is not appropriate, then it is likely that we will not observe particles in the energy range we measure.

\section{Synopsis}

There were two main purposes of this paper. First, to report on the existence of two distinct energetic electron populations of different origin in the Earth's magnetotail and second, to substantiate, in a more compact manner, the substorm model developed by Vogiatzis et al. (2005).

The comparison of electron data along and perpendicular to the magnetic field direction leaves no doubt that we are indeed dealing with two independent energetic electron populations being imbedded inside the plasma sheet. Moreover, having now a more complete substorm model, we are in the position to understand how these energetic electrons are generated and subsequently transported to the Cluster location. Yet the model appears capable of giving us answers regarding the ring current enhancement and the geosynchronous particle ejections during active geomagnetic periods. The fact that the magnetic field in the magnetotail does change significantly in the course of a substorm points to the crucial role of the locally induced electric field in the particle's energization; this electric field is an inseparable ingredient of dipolarization process. This latter fact puts into question studies which adopt a guiding center treatment, since at the times of substorms the magnetic field varies on the time scale of the particle's cyclotron turn. 
Acknowledgements. The authors would like to thank the Los Alamos team for providing the geosynchronous energetic particle data and CDAWeb for data access.

Topical Editor I. A. Daglis thanks two referees for their help in evaluating this paper.

\section{References}

Aggson, T. L., Heppner, J. P., and Maynard, N. C.: Observations of large magnetospheric electric fields during the onset phase of a substorm, J. Geophys. Res., 88, 3981-3990, 1983.

Akasofu, S.-I., DeForest, S., and McIlwain, C.: Auroral displays near the 'foot' of the fiels line of the ATS-5 satellite, Planet. Space Sci., 22, 25-28, 1974.

Angelopoulos, V., Kennel, C. F., Corotini, F. V., Pellat, R., Kivelson, M. G., Walker, R. J., Russell, C. T., Baumjohann, W., Feldman, W. C., and Gosling, J. T.: Statistical characteristics of bursty bulk flow eventes, J. Geophys. Res., 99, 21 257-21 280, 1994.

Angelopoulos, V., Corotini, F. V., Kennel, C. F., Kivelson, M. G., Walker, R. J., Russell, C. T., McPherron, R. L., Sanchez, E., Meng, C.-I., Baumjohann, W., Reeves, G. D., Belian, R. D., Sato, N., Friis-Christensen, E., Sutcliffe, P. R., Yumoto, K., and Harris, T.: Multipoint analysis of a bursty bulk flow event on April 11, 1985, J. Geophys. Res., 101, 4967-4989, 1996.

Baker, D. N.: Particle and field signatures of substorms in the near magnetotail, in: Magnetic Recconection in Space and Laboratory Plasmas, edited by: Hones, E. W., Geophys. Monogr. Ser., 30, 193-202, AGU, Washington, D.C., 1984.

Baker, D. N. and Stone, E. C.: Energetic electron anisotropies in the magnetotail: Identification of open and closed field lines, Geophys. Res. Lett., 3, 557-560, 1976.

Baker, D. N. and Stone, E. C.: Observations of energetic electrons $(E \geq 200 \mathrm{keV})$ in the Earth's magnetotail: Plasma sheet and fireball observations, J. Geophys. Res., 82, 1532-1546, 1977.

Baker, D. N., Kanekal, S. G., Li, X., Monk, S. P., Goldstein, J., and Burch, J. L.: An extreme distortion of the Van Allen belt arising from the 'Hallowe'en' solar storm in 2003, Nature, 432, 878-881, 2004.

Balogh, A., Dunlop, M. W., Cowley, S. W., et al.: The Cluster magnetic field investigation, Space Sci. Rev., 79, 65-91, 1997.

Baumjohann, W., Paschmann, G., and Luehr, H.: Characteristics of high-speed ion flows in the plasma sheet, J. Geophys. Res., 95, 3801-3809, 1990.

Baumjohann, W., Hesse, M., Kokubun, S., Mukai, T., Nagai, T., and Petrukovich, A. A.: Substorm dipolarization and recovery, J. Geophys. Res., 104, 24 995-25 000, 1999.

Birn, J. and Hesse, M.: Details of current disruption and diversion in simulations of magnetotail dynamics, J. Geophys. Res., 101, 15 345-15 358, 1996.

Birn, J., Thomsen, M. F., Borovsky, J. E., Reeves, G. D., McComas, D. J., and Belian, R. D.: Characteristic plasma properties during dispersionless substorm injections at geosynchronous orbit, J. Geophys. Res., 102, 2309-2324, 1997a.

Birn, J., Thomsen, M. F., Borovsky, J. E., Reeves, G. D., McComas, D. J., and Belian, R. D.: Substorm ion injections: Geosynchronous observations and test particle orbits in threedimensional dynamic MHD fields, J. Geophys. Res., 102, 23252341, $1997 b$.
Birn, J., Thomsen, M. F., Borovsky, J. E., Reeves, G. D., McComas, D. J., and Belian, R. D.: Substorm electron injections: Geosynchronous observations and test particle simulations, J. Geophys. Res., 103, 9235-9248, 1998.

Birn, J., Thomsen, M. F., Borovsky, J. E., Reeves, G. D., and Hesse, M.: Particle acceleration in the dynamic magnetotail, Physics of Plasmas, 7, 2149-2156, 2000.

Cravens, T. E.: Physics of Solar System Plasmas, Cambridge University Press, 1997.

Daglis, I. A. and Axford, W. I.: Fast ionospheric response to enhanced activity in geospace: Ion feeding of the inner magnetotail, J. Geophys. Res., 101, 5047-5065, 1996.

Daglis, I. A., Thorne, R. M., Baumjohann, W., and Orsini, S.: The terrestrial ring current: Origin, formation, and decay, Rev. Geophys., 37(4), 407-438, 1999.

Delcourt, D. C.: Particle acceleration by inductive electric fields in the inner magnetosphere, J. Atmos. Terr. Phys., 64, 551-559, 2002.

Delcourt, D. C. and Moore, T. E.: Precipitation of ions induced by magnetotail collapse, J. Geophys. Res., 97, 6405-6415, 1992.

Delcourt, D. C. and Sauvaud, J. A.: Plasma sheet ion energization during dipolarization events, J. Geophys. Res., 99, 97-108, 1994.

Delcourt, D. C., Sauvaud, J. A., and Pedersen, A.: Dynamics of single-particle orbits during substorm expansion phase, J. Geophys. Res., 95, 20 853-20 865, 1990.

Friedel, R. H. W., Korth, A., and Kremser, G.: Substorm onsets observed by CRRES: Determination of energetic particle source regions, J. Geophys. Res., 101, 13 137-13 154, 1996.

Gray, P. C. and Lee, L. C.: Particle pitch angle diffusion due to nonadiabatic effects in the plasma sheet, J. Geophys. Res., 87, 7445-7452, 1982.

Griffiths, D. J.: Introduction to Electrodynamics, Prentice-Hall, International, 2nd edn., 1999.

Hada, T., Nishida, A., Terasawa, T., and Hones, E. W.: Bidirectional electron pitch angle anisotropy in the plasma sheet, J. Geophys. Res., 86, 11 211-11 224, 1981.

Heikkila, W. J. and Pellinen, R. J.: Localized induced electric field within the magnetotail, J. Geophys. Res., 82, 1610-1614, 1977.

Hesse, M. and Birn, J.: On dipolarization and its relation to the substorm current wedge, J. Geophys. Res., 96, 19417-19426, 1991.

Hoshino, M., Mukai, T., Terasawa, T., and Shinohara, I.: Suprathermal electron acceleration in magnetic reconnection, J. Geophys. Res., 106, 25 979-25 997, 2001.

Jacquey, C. J., Sauvaud, J. A., and Dandouras, J.: Location and propagation of the magnetotail current disruption during substorm expansion: Analysis and simulation of an ISEE multionset event, Geophys. Res. Lett., 18, 389-392, 1991.

Kaufmann, R. L.: Substorm currents: Growth phase and onset, J. Geophys. Res., 92, 7471-7489, 1987.

Kennel, C. F. and Petschek, H. E.: Limits on stably trapped particle fluxes, J. Geophys. Res., 71, 1-28, 1966.

Klumpar, D. M.: Statistical distributions of the auroral electron albedo in the magnetosphere, in: Auroral Plasma Dynamics, pp. 163-171, AGU, Washington, D.C., 1993.

Klumpar, D. M., Quinn, J. M., and Shelley, E. G.: Counterstreaming electrons at the geomagnetic equator near $9 \mathrm{R}_{E}$, Geophys. Res. Lett., 15, 1295-1298, 1988. 
Korth, A., Pu, Z. Y., Kremser, G., and Roux, A.: A statistical study of substorm onset conditions at geostationary orbit, in: Magnetospheric Substorms, edited by: Kan, J. R., Potemra, T. A., Kokubun, S., and Iijima, T., Geophys. Monogr. Ser., 64, 343, AGU, Washington, D.C., 1991.

Lopez, R. E. and Lui, A. T. Y.: A multisatellite case study of the expansion of a substorm current wedge in the near-Earth magnetotail, J. Geophys. Res., 95, 8009-8017, 1990.

Lopez, R. E., Baker, D. N., Lui, A. T. Y., Sibeck, D. G., and Belian, R. D.: The radial and longitudinal propagation characteristics of substorm injections, Adv. Space Res., 8, (9)91-(9)95, 1988.

Lui, A. T. Y., Lopez, R. E., Krimigis, S. M., McEntire, R. W., and Zanetti, L. J.: A case study of magnetotail current sheet disruption and diversion, Geophys. Res. Lett., 15, 721-724, 1988.

Lui, A. T. Y., Mankofsky, A., Chang, C.-L., Papadopoulos, K., and Wu, C. S.: A current disruption mechanism in the neutral sheet: A possible trigger for substorm expansions, Geophys. Res. Lett., 17, 745-748, 1990.

Lui, A. T. Y., Chang, C.-L., Mankofsky, A., Wong, H.-K., and Winske, D.: A cross-field current instability for substorm expansions, J. Geophys. Res., 96, 11 389-11 401, 1991.

Nagai, T.: Observed magnetic substorm signatures at synchronous altitude, J. Geophys. Res., 87, 4405-4417, 1982.

Nagai, T., Fujimoto, M., Saito, Y., Machida, S., Terasawa, T., Nakamura, R., Yamamoto, T., Mukai, T., Nishida, A., and Kokubun, S.: Structure and dynamics of magnetic reconnection for substorm onsets with Geotail observations, J. Geophys. Res., 103, 4419-4440, 1998.

Rostoker, G., Kisabeth, J. L., Sharp, R. D., and Shelley, E. G.: The expansive phase of magnetospheric substorms 2. The response at synchronous altitude of particles of different energy ranges, J. Geophys. Res., 80, 3557-3570, 1975.

Sarris, E. T. and Axford, W. I.: Energetic protons near the plasma sheet boundary, Nature, 277, 460-462, 1979.
Sarris, E. T., Krimigis, S. M., Bostrom, C. O., Iijima, T., and Armstrong, T. P.: Location of the source of magnetospheric energetic particle bursts by multi-spacecraft observations, Geophys. Res. Lett., 3, 437-440, 1976.

Shepherd, G. G., Boström, R., Derblom, H., Fälthammar, C.-G., Gendrin, R., Kaila, K., Korth, A., Pedersen, A., Pellinen, R., and Wrenn, G.: Plasma and field signatures of poleward propagating auroral precipitation observed at the foot of the Geos 2 field line, J. Geophys. Res., 85, 4587-4601, 1980.

Shiokawa, K., Baumjohann, W., and Paschmann, G.: Bi-directional electrons in the near-Earth plasma sheet, Ann. Geophys., 21, 1497-1507, 2003.

Smets, R., Delcourt, D., Sauvaud, J. A., and Koperski, P.: Electron pitch angle distributions following the dipolarization phase of a substorm: Interball-Tail observations and modeling, J. Geophys. Res., 104, 14 571-14 581, 1999.

Speiser, T. W.: Particle trajectories in model current sheets, J. Geophys. Res., 70, 4219-4226, 1965.

Takahashi, K., Zanetti, L. J., McEntire, R. W., Potemra, T. A., and Lopez, R. E.: Disruption of the magnetotail current sheet observed by AMPTE/CCE, Geophys. Res. Lett., 14, 1019-1022, 1987.

Vogiatzis, I. I., Fritz, T. A., Zong, Q.-G., Baker, D. N., Sarris, E. T., and Daly, P. W.: Fine-time energetic electron behavior observed by Cluster/RAPID in the magnetotail associated with X-line formation and subsequent current disruption, Ann. Geophys., 23, 2265-2280, 2005.

West, H. I., Buck, R. M., and Kivelson, M. G.: On the configuration of the magnetotail near midnight during quiet and weakly disturbed periods: State of the magnetosphere, J. Geophys. Res., 83, 3805-3817, 1978.

Wilken, B., Axford, W. I., Daglis, I., et al.: RAPID: The imaging energetic particle spectrometer on Cluster, Space Sci. Rev., 79, 399-473, 1997.

Williams, D. J., Mitchell, D. G., Huang, C. Y., Frank, L. A., and Russell, C. T.: Particle acceleration during substorm growth and onset, Geophys. Res. Lett., 17, 587-590, 1990. 